\title{
Anterior Cingulate Cortex
}

National Cancer Institute

\section{Source}

National Cancer Institute. Anterior Cingulate Cortex. NCI Thesaurus. Code C139185.

The front region of the cingulate cortex that is located in the medial frontal lobe, adjacent to the corpus callosum. The anterior cingulate functions in decision making and in the regulation of emotions and physiological processes like blood pressure and heart rate. 